\title{
DISTÚRBIOS OSTEOMUSCULARES RELACIONADOS AO PROCESSO DE TRABALHO NO ATENDIMENTO PRÉ-HOSPITALAR
}

\author{
Rogério SAÇALA ${ }^{1}$ \\ Janayna do Rocio LUVIZOTTO ${ }^{2}$ \\ Gleidson Brandão OSELAME ${ }^{3}$ \\ Eduardo Borba NEVES ${ }^{4}$
}

\author{
${ }^{1}$ Enfermeiro. Centro Universitário Campos de Andrade. Curitiba - PR. \\ ${ }^{2}$ Docente do Curso de Enfermagem do Centro Universitário Campos de Andrade. Curitiba - PR. \\ ${ }^{3}$ Mestre em Engenharia Biomédica pela Universidade Tecnológica Federal do Paraná. Docente do Curso de Enfermagem do \\ Centro Universitário Campos de Andrade. Curitiba - PR. \\ ${ }^{4}$ Doutor em Saúde Pública. Docente do Centro Universitário Campos de Andrade. Curitiba - PR.
}

Recebido em: 29/06/2016 - Aprovado em: 30/11/2017 - Disponibilizado em: 30/12/2017

\begin{abstract}
RESUMO
Introdução: Os Distúrbios Osteomusculares Relacionados ao Trabalho (DORT) são lesões que acometem o sistema músculo esquelético de muitos trabalhadores, gerando o adoecimento destes, se não tratada preventivamente. O profissional que atua diariamente no atendimento pré-hospitalar apresenta-se vulnerável a esta patologia devido ao esforço físico associado às posturas inadequadas durante os atendimentos prestados à população. Objetivo: Identificar e quantificar os distúrbios osteomusculares que afetam os profissionais atuantes em um serviço de atendimento pré-hospitalar. Materiais e Métodos: Estudo quantitativo, com 20 profissionais do Serviço de Atendimento Móvel de Urgência (SAMU) que atuam na cidade de São José dos Pinhais -PR, Brasil. A coleta de dados foi realizada por meio do questionário Nordic Musculoskeletal Questionnaire (NMQ). Resultados e Conclusões: Observou-se que 95\% dos profissionais deste segmento apresentam dores no corpo. Do total da amostra, apena 15\% necessitaram de afastamento do trabalho por lesões osteomusculares, entretanto $75 \%$ já realizaram algum tipo de tratamento para diminuir os níveis de dor. Observou-se uma elevada frequência de dor na coluna lombar (70\% dos profissionais) e em outros seguimentos corporais. E ainda, $70 \%$ dos profissionais estudados apresentam níveis de dor superior a cinco (numa escala de zero a dez). Assim, pode-se concluir que esses profissionais estão expostos a uma sobrecarga física, vulnerabilidade de lesões, longos e exaustivos períodos de trabalho, gerando muitas vezes incapacidade parcial ou permanente para o trabalho.

Palavras-chave: Distúrbios Osteomusculares Relacionados ao Trabalho; Dor Osteomuscular; Atendimento de Emergência Pré-Hospitalar.

\section{RELATED MUSCULOSKELETAL DISORDERS WORK PROCESS PRE- HOSPITAL CARE}

\begin{abstract}
Introduction: Work-Related Musculoskeletal Disorders (WMSD) are injuries affecting the musculoskeletal system of many workers, generating the illness of these, if not treated preventively. The professional who works daily in the prehospital care has become vulnerable to this disease because of the physical effort associated with poor posture during the care provided to the population. Objective: To identify and quantify musculoskeletal disorders affecting professionals working in a pre-hospital care service. Methods: This was a quantitative study with 20 professional Mobile Emergency Service (SAMU) who work in the city of São José dos Pinhais-PR, Brazil. Data collection was performed using the Nordic Musculoskeletal Questionnaire (NMQ). Results and Conclusion: It was observed that 95\% present in this segment of the
\end{abstract}


professional body aches. Of the total sample, only $15 \%$ required sick leave for musculoskeletal injuries, however $75 \%$ have had some type of treatment to reduce pain levels. There was a high incidence of low back pain (70\% professionals) and other body segments. And yet, $70 \%$ of the studied professionals have higher pain levels to five (on a scale of zero to ten). Thus, it can be concluded that these workers are exposed to a physical overload vulnerable lesions, long and exhaustive work periods, and often causing partial or permanent disability to work.

Keywords: Work-Related Musculoskeletal Disorders; Musculoskeletal pain; Prehospital Emergency Care.

\section{INTRODUÇÃO}

Os distúrbios osteomusculares relacionados ao trabalho (DORT) são lesões que acometem músculos, tendões, nervos dos membros inferiores e superiores, regiões do pescoço e tronco(ULBRICHT et al., 2014). Estas lesões podem desencadear inflamações crônicas, com conseqüentes comprometimentos funcionais, principalmente, se forem decorrentes de movimentos repetitivos (MOREIRA e MENDES, 2005).

Neste contexto, o profissional que atua no atendimento pré-hospitalar exerce uma atividade que causa um grande desgaste físico, devido à forma com que se pode encontrar os mais diversos pacientes e seu grau de dependência, além das características do próprio ambiente de trabalho (LEITE, SILVA e MERIGHI, 2007).

Esses profissionais se deparam com inúmeras situações para prestar assistência à vitima de trauma, e precisam obrigatoriamente, se expor a diferentes ambientes naturais, enfrentar locais de difícil acesso, com presença de fogo, água, lama, energia elétrica sem controle, com risco muitas vezes para a própria vida (BARBOZA et al., 2008).

A exposição a estes ambientes são constantes e são intensificados pelo ritmo acelerado e muitas horas de trabalho que fazem parte da dinâmica de trabalho, o que acarreta diminuição na produtividade, bem como, um declínio na qualidade de vida(DE RAMALHO NETO et al., 2013). Outro fato que contribui para o comprometimento físico dos profissionais de saúde que atuam no pré hospitalar é que estes muitas vezes ignorarem o próprio desconforto físico para garantir a sobrevida do paciente vítima de trauma (LELIS et al., 2012).

Como consequência da exposição continua dos profissionais do seguimento préhospitalar, também pode ocorrer afastamento para tratamento de saúde e invalidez, faltas ao trabalho, o que acarreta na diminuição de funcionários e sobrecarga de trabalho para os demais (DE OLIVEIRA e PAIVA, 2013).

Por outro lado, ações da equipe multiprofissional que atua no atendimento préhospitalar podem contribuir na prevenção do aparecimento de lesões provenientes do 
trabalho, por meio da orientação e uso de técnicas posturais mais adequadas na execução de determinados movimentos durante o momento assistencial (TORRES, 2013).

Desta forma, o presente estudo teve como objetivo identificar e quantificar os distúrbios osteomusculares que afetam os profissionais atuantes em um serviço de atendimento pré-hospitalar.

\section{METODOLOGIA}

Tratou-se de uma pesquisa descritiva com análise quantitativa das variáveis envolvidas nos distúrbios osteomusculares apresentados pelos profissionais atuantes no atendimento pré-hospitalar.

A pesquisa foi realizada no Serviço de Atendimento Móvel de Urgência (SAMU), no município de São José dos Pinhais, estado do Paraná. A amostra foi composta por de $20 \%$ $(\mathrm{n}=20)$ de uma população total de 100 profissionais de saúde (enfermeiro, técnico e auxiliar de enfermagem e condutores de veículos de emergência) que atuam no atendimento pré-hospitalar.

Foram critérios de inclusão para a amostra: ser profissional de saúde do SAMU; atuar nas ambulâncias de suporte avançado, suporte básico e remoções simples no
Município; ter apresentado desconforto muscular em algum período da prestação de serviço; ter se afastado para tratamento de saúde relacionado a lesões ortopédicas e/ou estar com restrições devido o desenvolvimento de sintomas de DORT nos últimos 12 meses e sete dias precedentes à realização da pesquisa.

A coleta de dados foi realizada em agosto e setembro de 2014, por meio do questionário nórdico modificado Nordic Musculoskeletal Questionnaire (NMQ) (PEREIRA et al., 2014). O questionário é composto de 03 questões abertas e 17 fechadas, que buscam informações sobre a saúde e possíveis lesões, dores e desconforto durante o trabalho.

O estudo foi aprovado pelo Comitê de Ética em Pesquisa do Centro Universitário Campos de Andrade sob parecer consubstanciado $\mathrm{n}^{\circ} 0575$ de 2014. Os profissionais abordados assinaram o Termo de consentimento Livre Esclarecido (TCLE). Foram respeitados os aspectos éticos de pesquisas que envolvem Seres Humanos conforme a resolução 466/2012 do Conselho Nacional de Saúde. A análise dos dados foi realizada com base na distribuição de frequência absoluta e percentual das queixas, pelas diversas categorias criadas com base no perfil dos sujeitos estudados. 


\section{RESULTADOS}

Foram distribuídos 20 questionários aleatoriamente entre os profissionais que atuam nas ambulâncias: unidade de suporte avançado (USA), unidade de suporte básico (USB) e remoções simples. A Tabela 1 demonstra os resultados das variáveis analisadas.

Tabela 1. Perfil dos trabalhadores $(n=20)$ do Serviço de Atendimento Móvel de Urgência (SAMU), São José dos Pinhais, Paraná, 2014

\begin{tabular}{|c|c|c|}
\hline Variáveis & $\begin{array}{c}\text { Frequência } \\
\text { Absoluta na } \\
\text { Amostra }(\mathbf{n}=20)\end{array}$ & $\begin{array}{c}\text { Frequência } \\
\text { Relativa na } \\
\text { Amostra (\%) }\end{array}$ \\
\hline \multicolumn{3}{|l|}{ Idade } \\
\hline $20-30$ anos & 1 & 5 \\
\hline $31-40$ anos & 11 & 55 \\
\hline $41-50$ anos & 4 & 20 \\
\hline $51-60$ anos & 4 & 20 \\
\hline \multicolumn{3}{|l|}{ Sexo } \\
\hline Masculino & 13 & 65 \\
\hline Feminino & 7 & 35 \\
\hline \multicolumn{3}{|l|}{ IMC } \\
\hline Até 25.0 & 10 & 50 \\
\hline De 25.1 a 30.0 & 7 & 35 \\
\hline De 30.1 a 40.0 & 3 & 15 \\
\hline \multicolumn{3}{|l|}{ Função } \\
\hline Enfermeiro & 4 & 20 \\
\hline $\begin{array}{l}\text { Técnico de } \\
\text { enfermagem }\end{array}$ & 6 & 30 \\
\hline $\begin{array}{l}\text { Auxiliar de } \\
\text { enfermagem }\end{array}$ & 3 & 15 \\
\hline $\begin{array}{l}\text { Condutor } \\
\text { Socorrista }\end{array}$ & 7 & 35 \\
\hline $\begin{array}{l}\text { Tipo de } \\
\text { veículo }\end{array}$ & & \\
\hline Atendimento & 12 & 60 \\
\hline Remoções & 8 & 40 \\
\hline
\end{tabular}

Na percepção sobre local de trabalho, $85 \%(\mathrm{n}=17)$ dos profissionais descreveram ser suficiente a quantidade de funcionários atuando nas ambulâncias, contra $15 \%(\mathrm{n}=3)$, relatando a falta dos mesmos. Já no quesito treinamento em educação postural, $65 \% \quad(\mathrm{n}=13)$ dos entrevistados referem já ter participado de alguma orientação sobre o assunto.

Com relação às alterações físicas no trabalho, 20\% (n=4) da amostra não sabe o que é ergonomia e $80 \%(n=16)$ relata ter algum conhecimento sobre o assunto. Os profissionais referiram que durante atuação no serviço apresentavam dor no corpo $(95 \% ; \mathrm{n}=19)$. Ainda, $70 \% \quad(n=14)$ descreveram piora na qualidade de vida diária. A Tabela 2 apresenta os locais do corpo onde os profissionais referiram dor e seu respectivo nível.

Tabela 2. Locais do corpo e escala numérica da dor relativa aos dos trabalhadores $(n=20)$ do Serviço de Atendimento Móvel de Urgência (SAMU), São José dos Pinhais, Paraná, 2014.

\begin{tabular}{lcc}
\hline Variáveis & $\begin{array}{c}\text { Frequência } \\
\text { Absoluta } \\
\text { na Amostra } \\
(\mathbf{n = 2 0 )}\end{array}$ & $\begin{array}{c}\text { Frequência } \\
\text { relativa na } \\
\text { Amostra } \\
(\mathbf{\%})\end{array}$ \\
\hline Local do corpo & & \\
Pescoço & 2 & 10 \\
Ombro & 7 & 35 \\
Costas & 1 & 5 \\
Cotovelo & 1 & 5 \\
Antebraço & 0 & 0 \\
Lombar & 14 & 70 \\
Punho & 1 & 5 \\
Mãos & 1 & 5 \\
Dedos & 0 & 0 \\
MMII & 9 & 45
\end{tabular}


Escala da dor de 0 a 10

Dor de 1 a 4

Dor de 5 a 7

Dor de 7 a 10
Da amostra selecionada, somente $15 \%$ $(n=3)$ necessitou de afastamento do trabalho

\section{DISCUSSÃO}

Observou-se uma quantidade maior de profissionais do sexo masculino com média de idade de 35,5 anos, prevalecendo à função de condutores socorristas. Apenas 15\% $\quad(n=3)$ estavam com o IMC acima de 30, caracterizando obesidade. Neste sentido, conforme destaca Moreira e Mendes(MOREIRA e MENDES, 2005) o profissional com incapacidade de movimentação, ou seja, apresentando algum tipo de distúrbio relacionado a dificuldade em se movimentar é um profissional com grande chance de apresentar lesões futuras.

O maior número de profissionais $(60 \%$; $\mathrm{n}=12$ ) executam sua função nas ambulâncias do Serviço de Atendimento Móvel de Urgência (SAMU), onde o grau de dependência das vítimas são maiores e os locais para realização do atendimento são de difícil acesso, resultando em uma exigência maior sobre a condição física. Conforme apontam Barboza et $a l .,($ BARBOZA et al., 2008), as lesões tem propensão para o aparecimento quando os por lesões osteomusculares, enquanto que $75 \%$ $(n=15)$ já realizaram algum tipo de tratamento para melhora da dor.

movimentos são executadas com postura inadequada e associadas ao esforço físico.

A maior parte da amostra refere cansaço físico ao fim do plantão e uma grande utilização da força muscular, todavia, os profissionais relatam possuir força muscular suficiente para realização do trabalho. Neste sentido, Torres(TORRES, 2013) afirma que as maiores exigências musculares são em retirar e colocar o paciente do leito, mudá-lo de decúbito, transportá-lo na maca, cadeira de rodas e realizar higiene no leito.

Os profissionais $70 \% \quad(n=14)$ relatam realizar muita força na região lombar (70\%; $\mathrm{n}=14$ ). Desta forma, as lombalgias são a principal causa de afastamento dos profissionais da área da saúde com distúrbios osteomusculares (LELIS et al., 2012). Esses resultados estão de acordo com outros estudos que reportam que as dores musculares atualmente são consideradas como a maior causa de afastamentos das atividades laborais(VACARI et al., 2013) e que os principais motivos associados às lombalgias são provenientes de sobrecargas físicas e a má postura durante as atividades laborais, gerando 
de mecanismos de compressão dos discos intervertebrais e compressão da raiz nervosa(VACARI et al., 2013; ULBRICHT et al., 2014).

as atividades executadas. Autores apontam que essa percepção de autocuidado com sua postura, pode ser superestimada, pois para determinados grupos de trabalhadores, aceitar essa negligência seria expor vulnerabilidades que os mesmos não gostariam(NEVES e GOMIDE, 2007). Para melhorar este quadro, Barboza et al,(BARBOZA et al., 2008) afirma que os treinamentos com educação ergonômica podem prevenir os DORT, pois possuem um papel importante na saúde do profissional diminuindo a taxa de resistência a comunicação interna e aumentando a adesão a medidas profiláticas e preventivas.

Duarte, Souza e Macedo(DUARTE et al., 2012) apontam que os distúrbios osteomusculares podem se relacionar com a falta organizacional no trabalho, ou seja, aumento da jornada de trabalho, horas extras excessivas, ritmo acelerado e déficit de profissionais no setor. Desta forma, no presente estudo 85\% ( $\mathrm{n}=17)$ dos sujeitos apontaram ser suficiente o número de funcionários.

Relativo à variável treinamento em educação postural observou-se que houve uma quantidade maior de profissionais com conhecimento sobre o assunto 63\% ( $\mathrm{n}=17)$.
Entretanto, mais da metade dos trabalhadores $(60 \% ; n=12)$ afirmaram sempre estarem atentos a sua postura durante

Destaca-se, desta forma, que mesmo os profissionais recebendo treinamentos sobre esses assuntos, continuam apresentando lesões. De fato apresentar dores osteomusculares podem não relacionar-se ao ato de se machucar enquanto trabalha, mas é de extrema importância investigar a saúde física do trabalhador (SCHMIDT e DANTAS, 2012).

A maioria dos profissionais (95\%; $\mathrm{n}=19$ ) queixam-se de dor no corpo durante a atuação no serviço e $70 \%(\mathrm{n}=14)$ refere piora na qualidade de vida. Com estes aspectos um planejamento de práticas em atividades laborais e ergonômicas, seja antes ou durante o trabalho, objetivam relaxamento, diminuição da insatisfação e tensão durante o dia (UMANN, DE AZEVEDO GUIDO e DE OLIVEIRA FREITAS, 2011).

Os trabalhadores que declararam necessidade de afastamento do trabalho decorrente de DORT compreenderam 15\% $(n=3)$ e destes $75 \%(n=15)$ já havia realizado algum tipo de tratamento para minimizar a dor e demais sintomas. No estudo realizado por Mendonça et al.,(MENDONÇA et al., 2010) afirmam que uma boa postura associada a técnicas de relaxamento durante intervalos dos 
plantões e uma preparação física para as sobrecargas de trabalho, ajudam preventivamente nas lesões e dores por elas causadas.

Destacaram-se as regiões do corpo onde os profissionais referiram dor ou desconforto durante o trabalho ou permanente. Destacou-se a dor na coluna vertebral, especificamente a região lombar, atingindo $70 \%(n=14)$ e $45 \%(n=9)$ a dor nos membros inferiores. Resultado semelhante ao de Mendonça et al.,(MENDONÇA et al., 2010) As regiões de maior incidência de dor foram pescoço e costas superior

A dor no ombro esteve presente em $35 \%$ $(n=7)$ dos profissionais. Essa situação se deve possivelmente por se tratar de profissionais que executam suas atividades exigindo mais força dos membros superiores do que da região cervical. Já no estudo de Mendonça et $a l .,($ MENDONÇA et al., 2010) afirmam que os distúrbios músculo-esquelético são um conjunto de patologias inflamatórias que apresentam como sensação de peso e/ou fadiga, que acometem principalmente os membros superiores.

Relativo à intensidade da dor houve predominância $(50 \% ; n=10)$ com pontuação de dor de 5 a 7 pontos na escala numérica. Destaca-se que as sensações dolorosas de grau elevado estão pontualmente presentes entre os trabalhadores da área, sobretudo, quando relacionadas aos distúrbios osteomusculares (BARBOZA et al., 2008).

\section{CONCLUSÕES}

Observou-se que os profissionais que atuam no Atendimento pré-hospitalar, especificamente o Serviço de Atendimento Móvel de Urgência (SAMU), apresentam uma elevada frequência de dor na coluna lombar $(70 \%$ dos profissionais) e em outros seguimentos corporais. E ainda, $70 \%$ dos profissionais estudados apresentam níveis de dor superior a cinco (numa escala de zero a dez). Assim, pode-se concluir que esses profissionais estão expostos a uma sobrecarga física, vulnerabilidade de lesões, longos e exaustivos períodos de trabalho, gerando muitas vezes incapacidade parcial ou permanente para o trabalho.

\section{REFERÊNCIAS}

BARBOZA, M. C. N. et al. Doenças osteomusculares relacionadas ao trabalho (DORT) e sua associação com a enfermagem ocupacional. Revista Gaúcha de Enfermagem, v. 29, n. 4, p. 633, 2008. ISSN 1983-1447.

DE OLIVEIRA, A. C.; PAIVA, M. H. R. S. Prevalence and characteristics of accidents with biological materials involvin Ciência, Cuidado 
e Saúde, v. 12, n. 2, p. 323-330, 2013. ISSN 1984-7513.

DE RAMALHO NETO, $\mathrm{H}$. et al. Características do atendimento pré-hospitalar intradomiciliar em Curitiba-PR. Revista do Instituto de Ciencias da Saude (UNIP), v. 31, n. 2, p. 155-160, 2013.

DUARTE, A. F. et al. Fatores de riscos para distúrbios osteomusculares relacionados ao trabalho-DORT em profissionais de enfermagem. Revista de Pesquisa: Cuidado é fundamental online, n. Ed. Supl., p. 53-56, 2012. ISSN 2175-5361.

LEITE, P. C.; SILVA, A.; MERIGHI, M. A. B. A mulher trabalhadora de enfermagem e os distúrbios osteomusculares relacionados ao trabalho. Rev Esc Enferm USP, v. 41, n. 2, p. 287-91, 2007.

LELIS, C. M. et al. Distúrbios osteomusculares relacionados ao trabalho em profissionais de enfermagem: revisão integrativa da literatura. Acta Paul Enferm, v. 25, n. 3, p. 477-82, 2012.

MENDONÇA, D. S. et al. Incidência de Queixas Músculo-esqueléticas em Profissionais de Enfermagem de Unidades deTerapia Intensiva de Anápolis - Goiás - Brasil. Revista Brasileira de Ciências da Saúde, v. 13, n. 3, p. 69-76, 2010.

MOREIRA, A. M. R.; MENDES, R. Fatores de risco dos distúrbios osteomusculares relacionados ao trabalho de enfermagem. Rev. enferm. UERJ, v. 13, n. 1, p. 19-26, 2005.
PEREIRA, É. F. et al. Work-related stress and musculoskeletal complaints of orchestra musicians. Revista Dor, v. 15, n. 2, p. 112-116, 2014. ISSN 1806-0013.

SCHMIDT, D. R. C.; DANTAS, R. A. S. Qualidade de Vida no Trabalho e Distúrbios Osteomusculares Relacionados ao Trabalho entre profissionais de enfermagem. Acta Paul Enferm, v. 25, n. 5, p. 701-7, 2012.

TORRES, M. C. Riscos Ocupacionais do atendimento pré-hospitalar: uma revisão bibliográfica. Interfaces Científicas-Saúde e Ambiente, v. 1, n. 3, p. 69-77, 2013. ISSN 2316-3798.

ULBRICHT, L. et al. Prevalence of Workrelated Musculoskeletal Disorders (WMSD) symptoms among milkers in the State of Paraná, Brazil. In: AREZES, P. M. e (ORG.) (Ed.). Occupational Safety and Hygiene II. 1. London: CRC Press Taylor \& Francis Group, v.1, 2014. p.57-61. ISBN 1138001449.

UMANN, J.; DE AZEVEDO GUIDO, L.; DE OLIVEIRA FREITAS, E. Produção de conhecimento sobre saúde e doença na equipe de enfermagem na assistência hospitalar.

Ciência, Cuidado e Saúde, v. 10, n. 1, p. 162168, 2011. ISSN 1984-7513.

VACARI, D. A. et al. Principais métodos de diagnóstico postural da coluna lombar. Revista da Educação Física / UEM, v. 24, n. 2, p. 305315, 2013. ISSN 1983-3083. Disponível em: < http://www.scielo.br/scielo.php?script=sci_artte xt\&pid=S1983-30832013000200015\&nrm=iso $>$

NEVES, E. B.; GOMIDE, M. O uso de dispositivos de proteção auditiva nos tiros de fuzil e de artilharia. Cad. saúde colet.,(Rio J.), v. 15, n. 1, p. 97-116, 2007. ISSN 1414-462X. 